\title{
ELECTRO-OSMOTIC FLOW THROUGH CLOSED-OPEN-CLOSED MICROCHANNELS: AN APPROACH TO HYPHENATION OF CAPILLARY ELECTROPHORESIS AND MALDI
}

\author{
Varun Parmar $^{1}$, Theres Redeby ${ }^{2}$, Wouter van der Wijngaart ${ }^{1}$, Äsa Emmer ${ }^{2}$ and Göran Stemme ${ }^{1}$ \\ ${ }^{1}$ Microsystem Technology, School of Electrical Engineering, \\ ${ }^{2}$ Analytical Chemistry, School of Chemical Science and Engineering, \\ Royal Institute of Technology, Stockholm, SWEDEN. Email: wouter@s3.kth.se
}

\begin{abstract}
We suggest electro-osmotic driven flow (EOF) through closed-open-closed microchannels as a novel approach for spatial sample separation using capillary electrophoresis (CE) prior to matrix assisted laser desorption/ionization mass spectroscopy (MALDI-MS). For this purpose we built a system consisting of the series coupling of a closed fused silica capillary for separation, a microfabricated open microcanal for future MS detection and a second closed fused silica capillary for downstream liquid collection. This work verifies the EOF transport of a peptide sample in such a system with low dispersion.
\end{abstract}

\section{INTRODUCTION}

Capillary electrophoresis using EOF, coupled to mass spectrometry, is becoming a well-established technique for protein and peptide separation and characterisation. Electrospray Ionisation mass spectrometry (ESI-MS) is the technique most conveniently interfaced to $\mathrm{CE}$ since both these techniques are flow based [1]. Nevertheless, MALDIMS is a more tolerant technique towards contaminants and buffer additives than ESI-MS and produces mainly singly charged ions, a benefit when analyzing complex samples. However, hyphenation of CE and MALDI-MS is not uncomplicated, and microfluidics could provide interesting solutions for this purpose. Previously, off-line hyphenation of CE and MALDI-MS has been performed, which requires either continuous sample deposition onto a MALDI target [24] or CE fraction collection with subsequent MALDI-MS analysis [4-6].

Utilising miniaturised devices, surface tension enables controlled liquid flow in open microcanals [7] with direct physical access to the liquid for interfacing purposes. Such a microcanal can thus potentially function as a MALDI target. Previous efforts using microfluidics for MALDI-MS hyphenation include performing $\mathrm{CE}$ on-chip in completely open microcanals with subsequent off-line MALDI-MS by Liu et al. [8]. However, this technique suffers from suboptimal CE conditions. Brivio et al. [9] introduced online MALDI on microfluidic systems with "monitoring windows" for the inspection of reactions. This technique uses the
MALDI system vacuum for pressure driven flow (PDF) of the reagents and reaction products towards the monitoring window. However, this approach is not compatible with the EOF utilized in the CE.

In this work we suggest off-line CE-MALDI-MS hyphenation by combining the excellent CE properties of standard fused silica capillaries with the interfacing possibilities offered by open canal microfluidics. We specifically studied the electro-osmotic flow (EOF)-driven transport of a peptide sample through such a system.

\section{EXPERIMENTAL SETUP}

We built a system that consists of the series coupling of a closed inlet fused silica separation capillary, a microfabricated open microcanal for sample access and a closed outlet fused silica capillary for downstream liquid dispatch (Fig. 1).

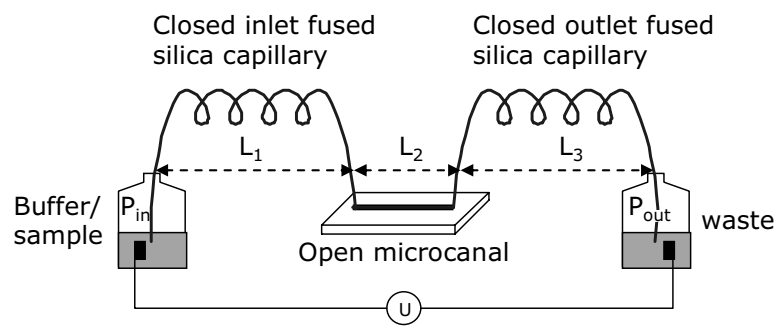

Figure 1. Schematic of the Closed-Open-Closed separation system. $L_{1,3} \approx 300-500 \mathrm{~mm} ; L_{2} \approx 10-30 \mathrm{~mm}$. The inlet pressure $P_{\text {in }}$ and outlet pressure $P_{\text {out }}$ are raised during liquid priming, and at atmospheric pressure during sample injection and separation. The separation voltage $U=10 \mathrm{kV}$.

The chips containing the microcanal were fabricated using subsequent photolithography, deep reactive ion etching (DRIE), oxidation and dicing of a silicon wafer. The microcanals used were $50 \mu \mathrm{m}$ deep and 25/50/100 $\mu \mathrm{m}$ wide, and had a length between 10 and $100 \mathrm{~mm}$.

The inlet and outlet fused silica capillaries had an inner diameter (ID) of $50 \mu \mathrm{m}$ and an outer diameter (OD) of 150 or $375 \mu \mathrm{m}$. They were coupled to the open microcanal by only bringing the capillaries perpendicularly to the chip so that contact between the fluid in the fused silica capillary and the open microcanal was established. We used syringe needles 
on a xyz-stage to guide the capillaries in place (Fig. 2). To avoid evaporation from the open canal during separation, the environment around the open microcanal was saturated with water vapour.

The inlet from the first fused silica capillary was manually positioned either in a buffer solution or in a sample reservoir. The outlet from the second fused silica capillary was positioned in a waste reservoir. The pneumatic pressure in each of the reservoirs $\left(\mathrm{P}_{\text {in }}\right.$ and $\left.\mathrm{P}_{\text {out }}\right)$ could be controlled externally. A voltage source of $10 \mathrm{kV}$ was coupled between electrodes in the reservoirs.

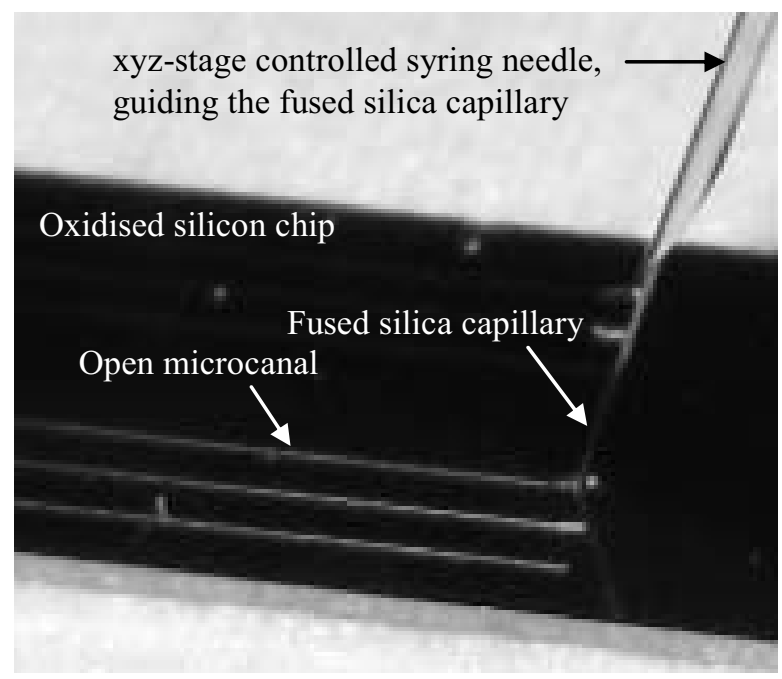

Figure 2. Close-up photograph of the fluidic coupling between a closed fused silica capillary and the open microcanal.

An electric field driven flow and separation can occur in all three fluidic channel sections. After electrophoretic separation in the first closed fused silica capillary, the sample enters the open microchannel section where it can be "dried" by solvent evaporation (similar to Liu et al., [8]), rendering a spatially separated sample for subsequent off-line MALDIMS.

A 206 PHD UVVIS detector from LINEAR (Reno, NV, USA) was used for online sample detection in the inlet and/or outlet capillary at position $\mathrm{L}_{\text {eff }}$ (Fig. 3). Flow speed can be determined by measuring the transport time of sample between the inlet capillary entry and the downstream detector position.

\section{EXPERIMENTAL RESULTS}

Both the buffer and the waste reservoirs were filled with 0.01 $\mathrm{M} \mathrm{NaH}{ }_{2} \mathrm{PO}_{4}, \mathrm{pH} 5.5$, as running buffer. At this $\mathrm{pH}$ the sample peptide Leucine-Enkephalin (Sigma-Aldrich, Stockholm, Sweden) is not charged and thus has no electrophoretic mobility and can be used to determine the EOF.

Liquid priming of the system was achieved by temporarily pressurising both buffer and waste reservoirs. Liquid filled the fused silica capillaries and entered the open microcanal.
Pressure at the reservoirs was removed once the liquid closed the electrical circuit. After priming, voltage was applied ( $\mathrm{U}=10 \mathrm{kV}$ in all measurements) over the system and a steady EOF was established.

The peptide sample $(1 \mathrm{mg} / \mathrm{ml}$ in water) was injected by switching off the voltage, manually moving the inlet of he inlet capillary to the sample reservoir, switching on the voltage for typically $20 \mathrm{~s}$, switching off the voltage again, moving back the inlet capillary to the buffer reservoir, and switching on the voltage again to start the separation.

A first set of measurements (Fig. 3) compares the EOF transport times between closed and closed-open systems in a number of subsequent runs with the same setup. The theoretically expected change in transport time between purely closed and closed-open systems is $\frac{t_{C, \text { theory }}}{t_{\text {CO,theory }}}=\frac{L_{1}}{L_{1}+L_{2}}=91.7 \%$. The measured change in transport time $\frac{\bar{t}_{C, \text { meas }}}{\bar{t}_{C O \text {, meas }}}=81 \% \pm 19 \%$. Thus, theory and measurement are in accordance.

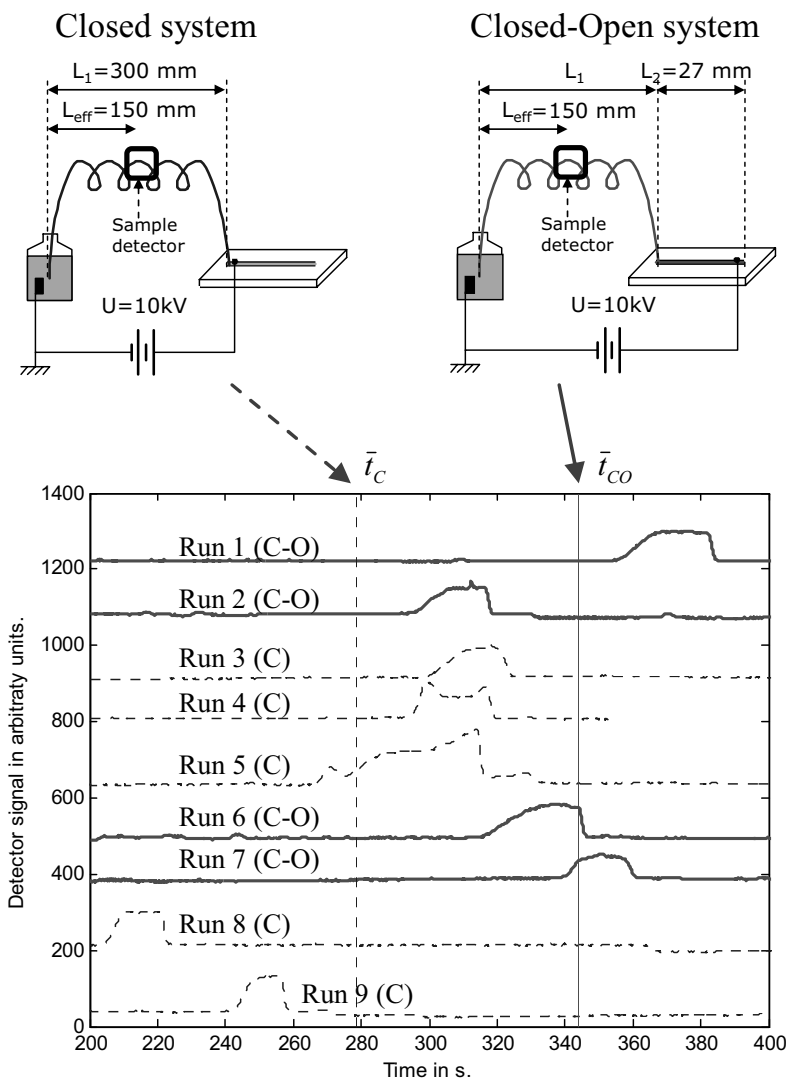

Figure 3. Detector signal for subsequent runs (1-9) in closed-open (red solid, index CO) and closed (blue dotted, index $C$ ) systems and their respective average transport time $t_{C O}$ and $t_{C}$ (vertical lines).

In a second set of experiments, peptide sample was successfully transported through a closed-open-closed 
system. In a first measurement setup (Fig. 4), both inlet and outlet capillary are guided through the same detector cell, allowing the same sample to be measured at two positions during the same run, as indicated in Fig. 4. However, the unconventional use of the detector causes mechanical stress in the capillaries, leading to their movement, causing severe baseline shifts in the plot. In this particular setup, the capillary OD was $375 \mu \mathrm{m}$, whereas the open microcanal width measured $50 \mu \mathrm{m}$. Undesired wetting at the contact between the capillary and the microcanal resulted in the formation of a liquid dead volume. It can clearly be observed that this causes broadening of the sample plug.

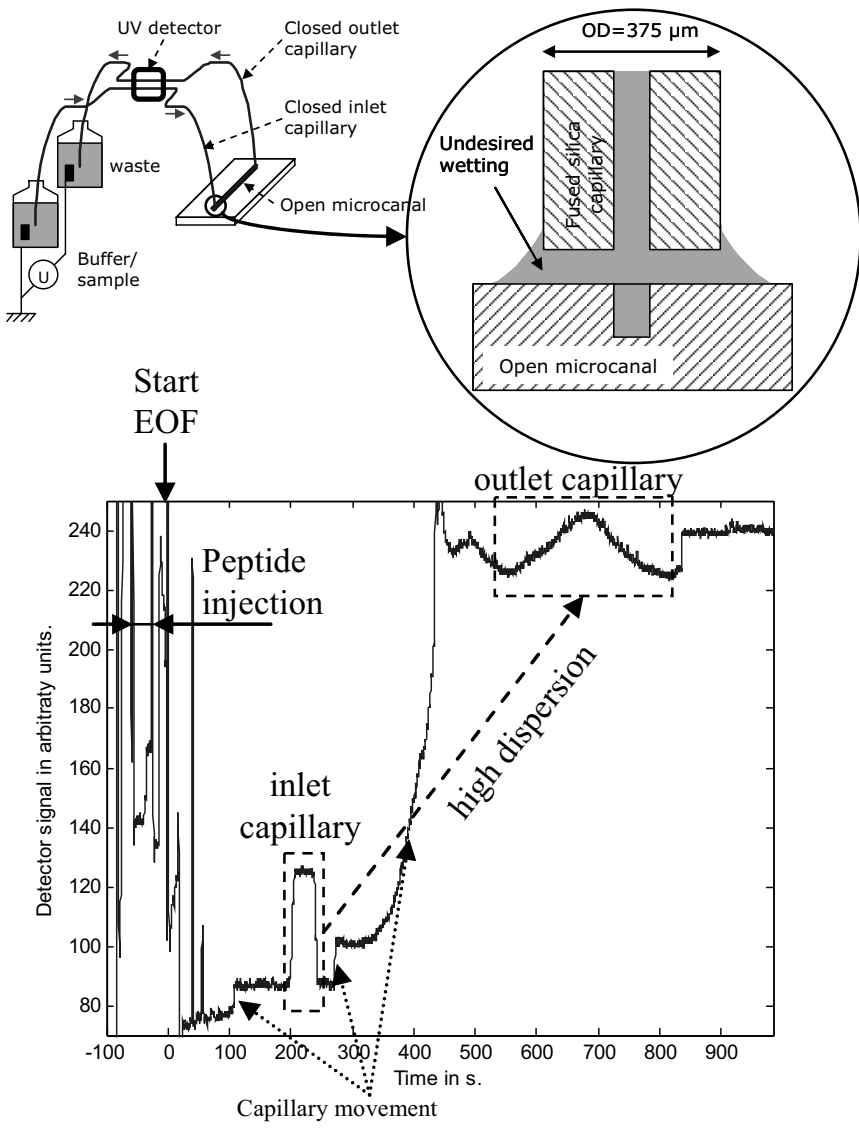

Figure 4. Measurement of peptide sample transport in Closed-Open-Closed channels with large OD silica capillaries. The setup allows detection of the sample in both inlet and outlet capillaries, but introduces signal shifting due to mechanical movement of the capillaries.

In a second measurement setup (Fig. 5), only the outlet capillary is guided through the UV detection cell. In this particular setup, capillaries with $\mathrm{OD}=150 \mu \mathrm{m}$ were used to reduce undesired liquid wetting around the fluidic coupling points. For both runs plotted in Fig. 5, sample dispersion in the system is limited. The pulse width of the signal (i.e. the time between the signal reaching $50 \%$ on the rising slope and $50 \%$ of the falling slope) increases from $17 \mathrm{~s}$ to $20 \mathrm{~s}$ in one run, and from $20 \mathrm{~s}$ to $28 \mathrm{~s}$ in a second run. The comparison between the dispersion observed in figures 4 and 5 respectively shows that proper fluidic coupling is crucial.

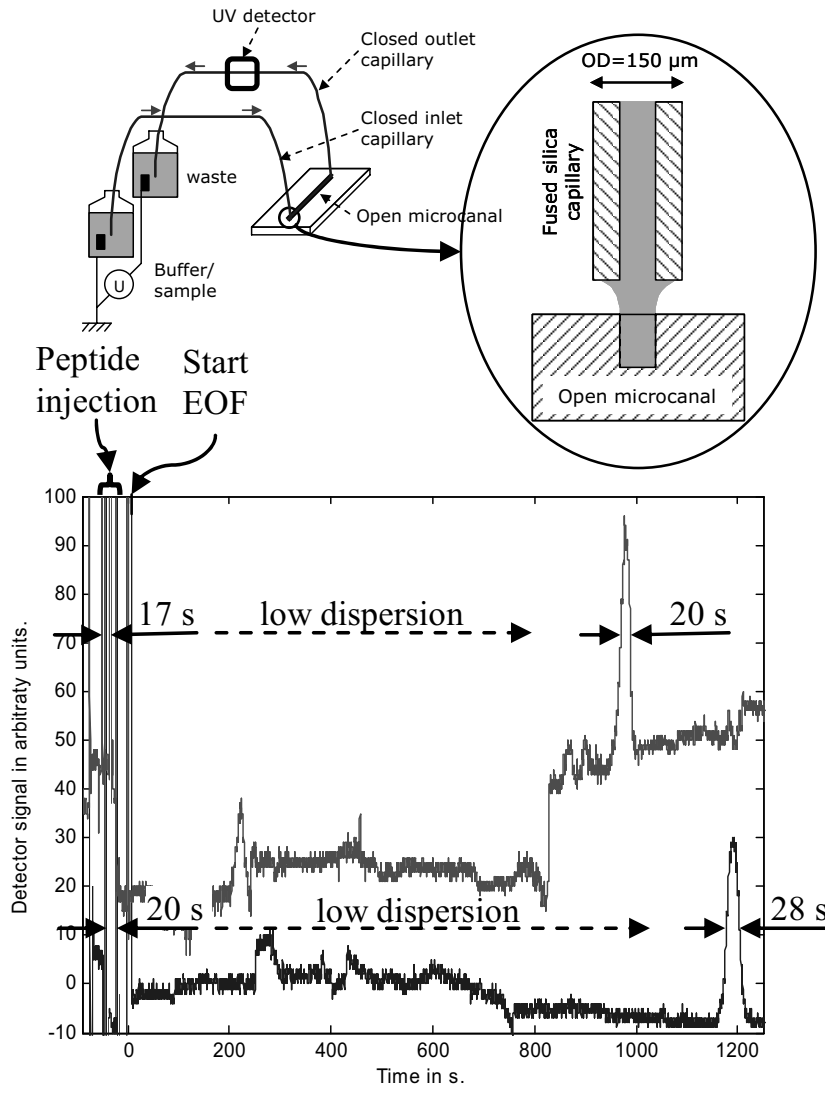

Figure 5. Measurement of two runs (plotted red/light grey and blue/dark grey) of peptide sample transport in ClosedOpen-Closed channels with small OD silica capillaries.

\section{CONCLUSIONS}

We suggested a novel approach for hyphenation of Capillary Electrophoresis and MALDI by serially coupling a closed fused silica separation capillary with an open microcanal. We verified that EOF driven peptide transport in closed-openclosed microfluidic systems can be achieved, and that a properly designed coupling of fused silica capillaries with open microcanals allows low sample dispersion. On-going work focuses on trimming the system for MALDI.

\section{REFERENCES}

[1] Stutz, "Advances in the analysis of proteins and peptides by capillary electrophoresis with matrix-assisted laser desorption/ionization and electrospray-mass spectrometry detection - Review", Electrophoresis, 26, 2005, pp. 1254-1290.

[2] H. Zhang and R.M. Caprioli, "Capillary Electrophoresis Combined with Matrix-Assisted Laser

Desorption/Ionization Mass Spectrometry; Continuous Sample Deposition on a Matrix-precoated Membrane Target", J. Mass Spectrom. 1996, 31, pp. 1039-1046.

[3] T. Rejtar, P.H. Hu, P. Juhasz, J. Campbell, M. Vestal, J. Preisler and B. Karger, "Off-Line Coupling of HighResolution Capillary Electrophoresis to MALDI-TOF 
and TOF/TOF MS", J. Proteome Res. 2002, 1, pp. 171179.

[4] P.A. van Veelen, U.R. Tjaden, J. van der Greef, A. Ingendoh and F. Hillenkamp, J. Chrom., "Off-line coupling of capillary electrophoresis with matrixassisted laser desorption mass spectrometry", 1993, 647, pp. 367-374.

[5] N. Ojima, T. Shingaki, T. Yamamoto and T. Masujima, "Droplet electrocoupling between capillary electrophoresis and matrix assisted laser desorption/ionization-time of flight-mass spectroscopy and its application", Electrophoresis 2001, 22, 34783482 .

[6] T. Johnson, J. Bergquist, R. Ekman, E. Nordhoff, M. Schurenberg, K.D. Kloppel, M. Muller, H. Lehrach and J. Gobom, " A CE-MALDI Interface Based on the Use of Prestructured Sample Supports", Anal. Chem. 2001, 73, pp. 1670-1675.

[7] J. Melin, W. van der Wijngaart and G. Stemme, "Behaviour and Design Considerations for Continuous Flow Closed-Open-Closed Liquid Microchannels", Lab on a Chip, 2005, 6, pp. 682-687.

[8] J. Liu, K. Tseng, B. Garcia, C. B. Lebrilla, E. Mukerjee, S. Collins and R. Smith, "Electrophoresis Separation in Open Microchannels. A Method for Coupling Electrophoresis with MALDI-MS", Anal. Chem. 2001, 73, pp. 2147-2151.

[9] M. Brivio, N. R. Tas, M. H. Goedbloed, H. J. G. E. Gardeniers, W. Verboom, A. van den Berg and D. N. Reinhoudt, "A MALDI-chip integrated system with a monitoring window", Lab on a Chip, 2005, 4, pp. 378381 . 